\title{
Condition for resonant optical bistability
}

\author{
A. V. Malyshev* \\ GISC, Departamento de Física de Materiales, Universidad Complutense, E-28040 Madrid, Spain
}

(Received 18 July 2011; published 27 December 2012)

\begin{abstract}
We address a two-level system in an environment interacting with an electromagnetic field in the dipole approximation. The resonant optical bistability induced by local-field effects is studied by considering the relationship between the population difference and the excitation field. The diversity of various systems is included by accounting for system self-action via the surface part of Green's dyadic in the general form. The bistability condition and the exact solution of the steady-state optical Bloch equations at the absolute bistability threshold are derived analytically.
\end{abstract}

DOI: 10.1103/PhysRevA.86.065804

PACS number(s): 42.65.Pc, 42.70.-a

Because of its underlying nature and possible applications in the field of all-optical processing, optical bistability (OB) has been a subject of intense experimental and theoretical research [1-29]. In early studies the use of a saturable absorber to induce OB was suggested [3-7]. The phenomenon was demonstrated experimentally for a cell of sodium vapor enclosed in a Fabry Perot interferometer and excited by a $\mathrm{cw}$ dyelaser [8]. Later it was conjectured that local-field corrections alone could give rise to mirrorless OB [9]. This type of bistability was extensively studied [10-14] and observed experimentally [15].

The practical interest in all-optical devices faded to some extent as their solid-state counterparts proved to perform better in terms of switching speed and device density. However, OB and optical hysteresis remain of considerable interest from the fundamental standpoint as a clear manifestation of a nonlinear light-matter interaction. Some new types of bistability mechanisms have been discussed recently [16-19]. Besides, the question of $\mathrm{OB}$ and hysteresis has received renewed attention in connection with novel hybrid zero-dimensional (0D) nanoscopic systems, e.g., artificial molecules comprising a semiconductor quantum dot (SQD) and metal nanoparticles (see Refs. [20-23] and references therein).

In this paper we address only mirrorless OB induced by local-field effects on a two-level system (TLS) interacting with an electromagnetic field in the dipole approximation. The local-field correction leads to a self-action of the system, which results in a nonlinear relation between the applied field and the one acting on the system. This type of OB mechanism can be relevant for a large variety of systems: dense 3D assemblies of two-level atoms [9,14], optically dense thin films of TLS [24,25] and films of linear molecular aggregates [26-28], hybrid metal-semiconductor systems [20-23], and a more general case of a TLS in an environment involving dielectric and conducting surfaces, such as a stratified medium, a microcavity, or a nanostructure.

OB can occur within a range of internal system parameters and external-field intensities; identifying these ranges is therefore an important problem and its analytical solution is desirable. To the best of the author's knowledge, so far

\footnotetext{
*on leave from A. F. Ioffe Physical-Technical Institute, 194021 St. Petersburg, Russia; a.malyshev@fis.ucm.es.
}

it has been solved exactly only for two particular cases. Friedberg et al. obtained analytically the bistability condition for the limiting case in which the active mechanism of the feedback is the nonlinear Lorentz shift of the resonance in a 3D gas of two-level atoms, which resulted from the near-field corrections [14]. Ignoring these corrections, Zakharov and Manykin derived the exact bistability criterion in the other limit in which the self-action is due to the radiated secondary field in a thin 2D film of TLS [25]. The case when both these fields contribute to the self-action has been studied only numerically so far [29].

We consider the simplest optical TLS coupled to an environment. The self-action of the TLS due to the environment can be described by the surface part of Green's dyadic evaluated at the position of the TLS. Therefore, in this case the nonlinearity is characterized by a complex number which should satisfy some condition for the $\mathrm{OB}$ to occur. Below, we derive this condition analytically.

Following Refs. [14,25,29], we address the OB condition by considering the relationship between the population difference and the excitation field within the framework of the Bloch equations for a $2 \times 2$ density matrix $\rho_{m n}(m, n=0,1)$ of a TLS. In the rotating-wave approximation these equations read

$$
\begin{aligned}
& \dot{Z}=-\gamma(Z+1)-\frac{1}{2}\left[\Omega P^{*}+\Omega^{*} P\right], \\
& \dot{P}=-(\Gamma+i \Delta) P+\Omega Z,
\end{aligned}
$$

where $Z=\rho_{11}-\rho_{00}$ is the population difference between the excited and the ground state of the TLS, $P$ is the amplitude of the off-diagonal density matrix element defined through $\rho_{10}=$ $-(i / 2) P \exp (-i \omega t), \gamma$ and $\Gamma$ are the relaxation constants of the population and the dipole moment, respectively, $\Delta=\omega_{0}-$ $\omega$ is the detuning of the driving field frequency $\omega$ from the TLS resonance $\omega_{0}$, and $\Omega=\mu \mathbf{E} / \hbar$ is the total electric field $\mathbf{E}$ (in frequency units) acting on the system, while $\boldsymbol{\mu}$ is the TLS optical transition dipole. The dipole moment of the system is $\mathbf{p}=-i P \boldsymbol{\mu}$, where $P$ is its complex amplitude.

To calculate the total field $\Omega$ the Maxwell's equations are to be solved for a particular geometry. However, for a single dipole in an environment the total field $\Omega$ can be represented in the general form

$$
\Omega=\widetilde{\Omega}_{0}+\Omega_{2}, \quad \Omega_{2}=-i G P
$$


where $\widetilde{\Omega}_{0}$ is the renormalized incident field and $\Omega_{2}$ is the secondary dipole field. The latter field is due to the feedback of the environment and is related to the corresponding response function [30-32]. It is determined by the surface part of Green's dyadic evaluated at the position of the dipole. The near-zone component of the secondary dipole field, governed by $\operatorname{Re}(G)$, can originate from the near-field corrections [14], while the far-zone contribution, governed by $\operatorname{Im}(G)$, can be due to radiation [25]. In hybrid nanosystems comprising an SQD, the feedback is provided by the secondary reflected field of the optical transition dipole moment. Thus, independently of its physical mechanism, the self-action is determined by the single complex-valued parameter $G$. The total field $\Omega$ acting on the TLS therefore depends on its state, which can give rise to a variety of effects, such as OB.

Under the steady-state conditions Eqs. (1a) and (1b) read

$$
\begin{aligned}
\frac{\left|\widetilde{\Omega}_{0}\right|^{2}}{\gamma \Gamma} & =-\frac{Z+1}{Z} \frac{\left(\Gamma-G_{\mathrm{I}} Z\right)^{2}+\left(\Delta+G_{\mathrm{R}} Z\right)^{2}}{\Gamma^{2}}, \\
P & =\frac{Z \widetilde{\Omega}_{0}}{\left(\Gamma-G_{\mathrm{I}} Z\right)+i\left(\Delta+G_{\mathrm{R}} Z\right)},
\end{aligned}
$$

where $G_{\mathrm{R}}=\operatorname{Re}(G)$ and $G_{\mathrm{I}}=\operatorname{Im}(G)$. The above equations highlight a known result of cavity quantum electrodynamics: the real part of Green's dyadic shifts the frequency, while the imaginary part renormalizes the decay rate [30-32]. Both effects can be active mechanisms of OB.

Equation (3a) is of the third order in $Z$ and can therefore have three real roots for some values of $\Delta, \Gamma, G_{\mathrm{R}}$, and $G_{\mathrm{I}}$ ( $\Gamma$ is used as a unit rate henceforth). These three solutions are different when the right-hand side of Eq. (3a) has a minimum and a maximum (see the solid line in Fig. 1), corresponding to two real roots of the right-hand-side derivative, which satisfy the following equation:

$$
2|G|^{2} Z^{3}+\left(|G|^{2}+2 G_{\mathrm{R}} \Delta-2 G_{\mathrm{I}}\right) Z^{2}-\left(1+\Delta^{2}\right)=0 .
$$

A threshold for bistability occurs when Eq. (4) has a double root (merged extrema; see the dashed or dotted lines in Fig. 1). In this case the solutions of Eq. (3a) are

$$
Z_{1}=Z_{2}=-\frac{|G|^{2}+2\left(G_{\mathrm{R}} \Delta-G_{\mathrm{I}}\right)}{3|G|^{2}}, \quad Z_{3}=-\frac{Z_{1}}{2} .
$$

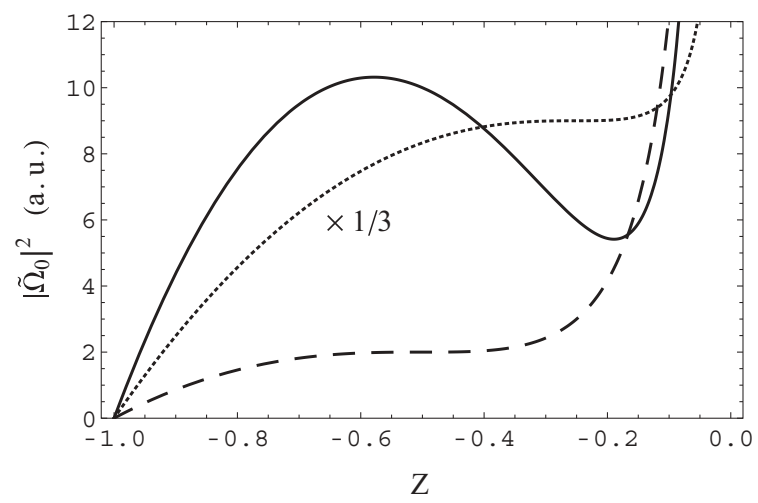

FIG. 1. The right-hand side of Eq. (3a) for $G=6, \Delta=1$ (solid line), $G=4, \Delta=1$ (dashed line), and $G=8 i, \Delta=0$ (dotted line).
Using the above root $Z_{1,2}$ and the condition $-1 \leqslant Z \leqslant 0$, one obtains an important constraint for the detuning $\Delta$ :

$$
G_{\mathrm{R}} \Delta-G_{\mathrm{I}} \leqslant|G|^{2}
$$

The formal condition of the existence of the double root, (5), is determined by the following equation on $\Delta$ :

$$
\left(|G|^{2}+2 G_{\mathrm{R}} \Delta-2 G_{\mathrm{I}}\right)^{3}-27|G|^{4}\left(1+\Delta^{2}\right)=0,
$$

which is cubic in $\Delta$ and can also have three real solutions. It can be shown that only two of them, say $\Delta_{1}<\Delta_{2}$, satisfy constraint (6) and yield the upper and lower threshold values of the population difference $Z$ and the external field $\widetilde{\Omega}_{0}$. Therefore, Eq. (4) has three different real roots within a window of detunings $\Delta_{1} \leqslant \Delta \leqslant \Delta_{2}$. The absolute bistability threshold occurs when the window shrinks to a point, i.e., when a degenerate root $\Delta_{1}=\Delta_{2}$ of Eq. (7) appears. After some algebra, one can obtain the following alternative for the existence of such a double root:

$$
\begin{aligned}
G_{\mathrm{R}}^{4}+2\left(G_{\mathrm{I}}^{2}-4 G_{\mathrm{I}}-8\right) G_{\mathrm{R}}^{2}+G_{\mathrm{I}}^{3}\left(G_{\mathrm{I}}-8\right) & =0, \\
G_{\mathrm{I}}+1 & =0 .
\end{aligned}
$$

Only two of the solutions of the biquadratic equation (8a), satisfy constraint (6). Analyzing the roots of Eq. (4) in the vicinity of the solution to Eqs. (7) and (8b), one finds that the system can be bistable below the line given by $G_{\mathrm{I}}=-1$, while being critical (i.e., $Z_{1,2}=-1$ ) on the line for $\left|G_{R}\right| \leqslant \sqrt{3}$. Finally, the absolute bistability threshold is

$$
\begin{aligned}
G_{\mathrm{R}}^{2} & =8+4 G_{\mathrm{I}}-G_{\mathrm{I}}^{2}+8 \sqrt{1+G_{\mathrm{I}}}, \quad-1<G_{\mathrm{I}} \leqslant 8 \\
-\sqrt{3} & \leqslant G_{\mathrm{R}} \leqslant \sqrt{3}, \quad G_{\mathrm{I}}=-1 .
\end{aligned}
$$

For completeness, we also provide the expression for the corresponding detuning:

$$
\Delta=\frac{|G|^{4}-4 G_{\mathrm{R}}^{2}\left(G_{\mathrm{I}}+3\right)-4 G_{\mathrm{I}}^{2}\left(G_{\mathrm{I}}-1\right)}{|G|^{4}+8 G_{\mathrm{I}}\left[G_{\mathrm{I}}^{3}+G_{\mathrm{R}}^{2}\left(G_{\mathrm{I}}+2\right)\right]} G_{\mathrm{R}} .
$$

Equation (1a) provides the condition for the existence of the three real roots of Eq. (3a). We studied the stability of these by analyzing Lyapunov exponents of Eq. (1a) in the vicinity of a stationary solution and calculated the stability phase map numerically. Figure 2 shows this phase diagram in the space of the feedback parameter $G$. Three different real solutions of Eq. (3a) exist within the white area, two of them being stable. The line dividing the two phases represents the analytical condition, Eq. (1a), which gives also the bistability threshold. The general criterion, Eq. (1a), recovers both reported exact results: $G_{\mathrm{I}}>8$ for $G_{\mathrm{R}}=0$ [25] and $G_{\mathrm{R}}>4$ for $G_{\mathrm{I}}=0$ [14] (the corresponding threshold values of $G$ are marked by filled circles in Fig. 2). Note the mirror symmetry of the bistability map with respect to the line $G_{\mathrm{R}}=0$, which reflects the invariance of Eq. (3a) under the simultaneous change of signs of $G_{\mathrm{R}}$ and $\Delta$ [29].

Equations (5) and (10) give the full solution of the steady-state problem at the absolute bistability threshold, while Eq. (1a) is the bistability condition, which constitutes the main result of the paper.

Finally, we note that novel hybrid plasmonic nanosystems, such as an SQD and a metal nanoparticle complex (see Ref. [23] and references therein) or an SQD embedded in a stratified medium, are excellent model systems with adjustable 


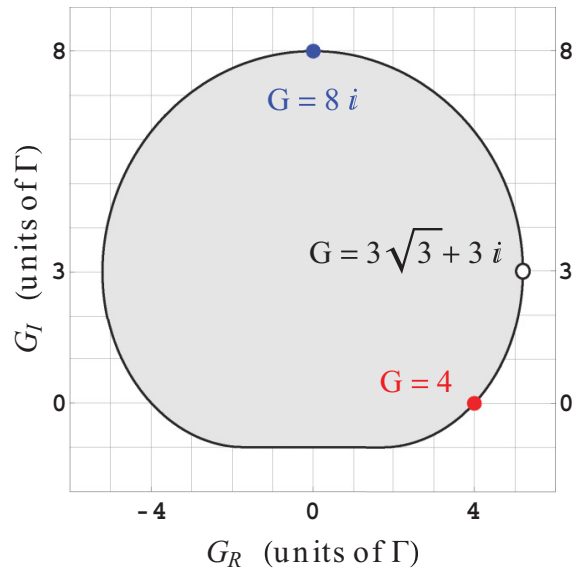

FIG. 2. (Color online) Stability phase map: outside the gray area the system can be bistable. The solid line represents the phase boundary given by Eq. (1a). See text for more details.

nonlinearity. The self-action field in these complexes is the secondary reflected field of the SQD optical transition dipole moment acting back on the SQD. In the most general case such environment feedback results in a complex selfaction parameter $G$, which can be engineered by an appropriate choice of materials, geometry, and/or external control parameters. Thus, if the SQD transition frequency is far from the plasmon resonance of the system, then, typically, $\left|G_{\mathrm{R}}\right| \gg\left|G_{\mathrm{I}}\right|$ and the dominant mechanism of the bistability is the nonlinear Lorentz shift [14]. If the excitation frequency is near the surface plasmon resonance, then the feedback can be almost purely imaginary: $\left|G_{\mathrm{I}}\right| \gg\left|G_{\mathrm{R}}\right|$, with $G_{\mathrm{I}}>0$. Algebraically, this case is equivalent to the one of radiation-induced self-action [25]. A very interesting case is $G_{\mathrm{I}}<0$, which can hardly be realized in the traditional extended 3D or 2D systems of two-level atoms. Nanoscopic hybrids are much more promising from this point of view due to their greater diversity. In the latter case various instabilities such as auto-oscillations can be expected under steady-state excitation. However, the detailed study of these instabilities goes beyond the scope of the present contribution and will be analyzed elsewhere.

Summarizing, we have addressed the mirrorless resonant OB of a TLS in an environment by considering the relationship between the population difference and the intensity of the excitation field. The feedback of the environment was introduced in a general form via the surface part of Green's dyadic. We derived the analytical bistability condition and obtained the exact steady-state solution of the Maxwell-Bloch equations at the absolute bistability threshold. Our findings open the possibility of easily analyzing diverse physical systems, determining the experimental conditions (such as the appropriate detuning from the bare resonance and the intensity of the external field) necessary to observe bistability and other nonlinear optical effects, and designing and engineering new systems with desirable nonlinear optical properties.

Support from the project MOSAICO (FIS2006-01485), fruitful discussions with V. A. Malyshev, and the hospitality of the University of Groningen are gratefully acknowledged.
[1] E. Abraham and S. D. Smith, Rep. Prog. Phys. 45, 815 (1982).

[2] H. M. Gibbs, Optical Bistability: Controlling Light with Light (Academic Press, New York, 1985).

[3] A. Szöke, V. Danen, J. Goldhar, and N. A. Kurnit, Appl. Phys. Lett. 15, 376 (1969).

[4] J. W. Austin and L. G. DeShazer, Program of the 1971 Spring Meeting of the Optical Society of America, J. Opt. Soc. Am. 61, 648-697 (1971).

[5] E. Spiller, Program of the 1971 Spring Meeting of the Optical Society of America, J. Opt. Soc. Am. 61, 648-697 (1971).

[6] S. L. McCall, Phys. Rev. A 9, 1515 (1974).

[7] R. Bonifacio and L. A. Lugiato, Opt. Commun. 19, 172 (1976).

[8] H. M. Gibbs, S. L. McCall, and T. N. C. Venkatesan, Phys. Rev. Lett. 36, 1135 (1976).

[9] C. M. Bowden and C. C. Sung, Phys. Rev. A 19, 2392 (1979).

[10] I. Abram and A. Maruani, Phys. Rev. B 26, 4759 (1982).

[11] F. A. Hopf, C. M. Bowden, and W. H. Louisell, Phys. Rev. A 29, 2591 (1984).

[12] Y. Ben-Aryeh, C. M. Bowden, and J. C. Englund, Phys. Rev. A 34, 3917 (1986).

[13] M. E. Crenshaw, M. Scalora, and C. M. Bowden, Phys. Rev. Lett. 68, 911 (1992).

[14] R. Friedberg, S. R. Hartmann, and J. T. Manassah, Phys. Rev. A 39, 3444 (1989).

[15] M. P. Hehlen, H. U. Güdel, Q. Shu, J. Rai, S. Rai, and S. C. Rand, Phys. Rev. Lett. 73, 1103 (1994).
[16] A. A. Afanas'ev, A. G. Cherstvy, R. A. Vlasov, and V. M. Volkov, Phys. Rev. A 60, 1523 (1999).

[17] A. E. Kaplan and S. N. Volkov, Phys. Rev. Lett. 101, 133902 (2008).

[18] A. E. Kaplan and S. N. Volkov, Phys. Rev. A 79, 053834 (2009).

[19] S. N. Volkov and A. E. Kaplan, Phys. Rev. A 81, 043801 (2010).

[20] W. Zhang, A. O. Govorov, and G. W. Bryant, Phys. Rev. Lett. 97, 146804 (2006).

[21] R. D. Artuso and G. V. Bryant, Nano Lett. 8, 2106 (2008).

[22] S. M. Sadeghi, Nanotechnology 21, 455401 (2010).

[23] A. V. Malyshev and V. A. Malyshev, Phys. Rev. B 84, 035314 (2011).

[24] M. G. Benedict, V. A. Malyshev, E. D. Trifonov, and A. I. Zaitsev, Phys. Rev. A 43, 3845 (1991).

[25] S. M. Zakharov and E. A. Manykin, Poverkhnost' 2, 137 (1988).

[26] V. Malyshev and P. Moreno, Phys. Rev. A 53, 416 (1996).

[27] J. A. Klugkist, V. A. Malyshev, and J. Knoester, J. Chem. Phys. 127, 164705 (2007).

[28] J. A. Klugkist, V. A. Malyshev, and J. Knoester, J. Chem. Phys. 128, 084706 (2008).

[29] A. N. Orayevsky, D. J. Jones, and D. K. Bandy, Opt. Commun. 111, 163 (1994).

[30] G. S. Agarwal, Phys. Rev. A 11, 230 (1975).

[31] G. S. Agarwal, Phys. Rev. A 12, 1475 (1975).

[32] G. S. Agarwal and S. D. Gupta, Phys. Rev. A 57, 667 (1998). 